EUROPEAN JOURNAL OF PURE AND APPLIED MATHEMATICS

Vol. 14, No. 4, 2021, 1169-1183

ISSN 1307-5543 - ejpam.com

Published by New York Business Global

\title{
Denjoy-type Integrals in Locally Convex Topological Vector Space
}

Rodolfo E. Maza ${ }^{1, *}$, Sergio R. Canoy, Jr.

${ }^{1}$ Department of Mathematics and Statistics, College of Science and Mathematics, MSU-IIT, Iligan City 9200, Philippines

\begin{abstract}
In this paper, we introduce $A C^{*}$ and $A C G^{*}$-type properties and then, using these conditions along with other concepts, define two Denjoy-type integrals of a function with values in a locally convex topological vector space (LCTVS). We show, among others, that these newly defined integrals are included in the $S H$ integral, a version of the Henstock integral, for LCTVSvalued functions.
\end{abstract}

2020 Mathematics Subject Classifications: 46G12, 46G05, 28B05

Key Words and Phrases: $A C^{*}, A C G^{*}$, Denjoy integral, weak Denjoy integral, $S H$ integral

\section{Introduction}

The Henstock-Kurzweil $(H K)$ integral, developed independently by Ralph Henstock and Jaroslav Kurzweil, is known to generalize the Lebesgue integral. This integral uses partitions called $\delta$-fine partitions in its definition making it a Riemann-type integral. Thus, the $H K$ integral is much simpler to deal with than the Lebesgue integral which requires a considerable understanding of measure theory to fully grasp its definition.

In the real-valued case, the $H K$ integral is known to satisfy Henstock's lemma [15]. However, this property does not necessarily hold in the Banach-valued case (see [2]). Thus, for non-real-valued functions, some stronger versions of the $H K$ integral had been introduced. In [2], Cao defined the $H L$ integral for Banach-valued functions. Paluga and Canoy [9] introduced the Henstock-Kurzweil $(H K)$ and the $S H$ integrals for functions taking values in a topological vector space. From their respective definitions, it is clear that for functions taking values in a locally convex topological vector spaces, the family of $S H$ integrable functions is contained in the family of $H K$ integrable functions (see [9]). For functions with values in a locally convex topological vector space (LCTVS), Maza et al. [6] recently defined an $S L$-type integral (an integral which uses a Strong-Lusin-type condition) and showed that this integral is equivalent to the $S H$ integral.

${ }^{*}$ Corresponding author.

DOI: https://doi.org/10.29020/nybg.ejpam.v14i4.4115

Email addresses: rodolfo.maza@g.msuiit.edu.ph (R. Maza), sergio.canoy@g.msuit.edu.ph (S. Canoy) 
Other concepts that also played important roles in integration theory are the $A C^{*}$ and $A C G^{*}$ properties. The notion of $A C G^{*}$ dates back to Lusin and Khintchine ([5], [4]). Alternative definitions of $A C^{*}$ and $A C G^{*}$ are given by Lee and Vyborney in [16] and [14], where they used this slightly modified definition of $A C G^{*}$ to characterize the $H K$ integral. More specifically, they showed that a function $f:[a, b] \rightarrow \mathbb{R}$ is $H K$-integrable on $[a, b]$ if and only if there exists an $A C G^{*}$-function on $[a, b]$ such that $F^{\prime}(t)=f(t)$ almost everywhere. In 1995, following Lee's alternative definitions of $A C^{*}$ and $A C G^{*}$, Canoy and Navarro [1] defined a Denjoy-type integral for Banach-valued functions and in 1998 Skvortsov and Solodov [12] adopted such definition and called that integral the Denjoy-Bochner integral. In this paper, following these earlier definitions, we introduce $A C^{*}$ and $A C G^{*}$-type properties for LCTVS-valued functions and, subsequently, define two Denjoy-type integrals. Further, we show among others, that one of these integrals is included in the other and that both are included in the $S H$-integral. It is shown that for functions taking values in a Banach space, every Denjoy-Bochner integrable function is Denjoy integrable.

Recall that a topological vector space $X$ is a real vector space together with a Hausdorff topology $\tau$ such that the scalar multiplication and the vector addition associated with $X$ are continuous with respect to $\tau$ (see [10]). Continuity of the vector addition would then imply that for every open set $U$, there are open sets $V_{1}$ and $V_{2}$ such that $V_{1}+V_{2} \subseteq U$. More generally, for every $\theta$-nbd $U$ (an open set containing the zero vector $\theta$ of $X$ ) and $n \in \mathbb{N}$ there are $\theta$-nbds $V_{1}, V_{2}, \ldots, V_{n}$ such that $V_{1}+V_{2}+\cdots+V_{n} \subseteq U$ (see [3] and [10]). Given two topological spaces $X$ and $Y$, a function $F: X \rightarrow Y$ is continuous if $F^{-1}(U)$ is open in $X$ whenever $U$ is open in $Y$ [3].

A set $A \subseteq X$, where $X$ is a topological vector space, is absorbing if for every $x \in X$, there exists $t>0$ such that $x \in t A$; it is convex if for every $x, y \in A$ and $0 \leq t \leq 1$, $t x+(1-t) y \in A$; it is balanced if $\alpha A \subseteq A$ for every $|\alpha| \leq 1$. A sequence $\left\langle r_{i}\right\rangle_{i=1}^{n}$ of positive real numbers is unitary if $\sum_{i=1}^{n} r_{i}=1$. A set $A$ is convex if for every unitary sequence $\left\langle r_{i}\right\rangle_{i=1}^{n}$, we have $\sum_{i=1}^{n}\left(r_{i} A\right) \subseteq A$. A topological vector space $X$ is said to be locally convex if there is a local base consisting of convex sets in $X$. It is known that every locally convex topological vector space has a local base at $\theta$ consisting of absorbing, balanced, and convex sets.

A function $\rho: X \rightarrow \mathbb{R}$ is a seminorm if for all $u, v \in X$ and $k \in \mathbb{R}$, we have $(i)$ (sub-additivity) $\rho(u+v) \leq \rho(u)+\rho(v)$ and (ii) (absolute homogene.ity) $\rho(k u)=|k| \rho(u)$. A family of seminorms $\left\{\rho_{\alpha}\right\}_{\alpha}$ is called separated (or separating) if whenever $\rho_{\alpha}(x)=0$ holds for all $\alpha$, then $x$ is necessarily the zero vector $\theta$.

For a given absorbing set $A \subseteq X$, the Minkowski functional of $A$ on $X$ is defined by $\Phi_{A}(x)=\inf \{\lambda>0: x \in \lambda A\}$ for every $x \in X$. If $U \subseteq X$ is a balanced, absorbing and convex set, then $U=\left\{x \in X: \Phi_{U}(x)<1\right\}$ and $\Phi_{U}$ is a semi-norm on $X$. Also, for any $V \subseteq X, \Phi_{r V}(x)=\frac{1}{r} \Phi_{V}(x)$ for all positive real numbers $r$ and $x \in X$ (see [11]). For any given absorbing sets $A$ and $B$ with $A \subseteq B \subseteq X, \Phi_{B}(t) \leq \Phi_{A}(t)$ for all $t \in X$. One may refer to [10] for the definition, the earlier mentioned results, and a detailed discussion of the Minkowski functional.

A function $\delta:[a, b] \rightarrow \mathbb{R}^{+}$is called a gauge [14]. A finite collection $D=\left\{\left[u_{i}, v_{i}\right]\right.$ : 
$1 \leq i \leq n\}$ of non-overlapping closed sub-intervals of $[a, b]$ is called a partial partition of $[a, b]$. If the union of the intervals in $D$ is equal to $[a, b]$, then $D$ is called partition of $[a, b]$. A finite collection of ordered pairs $\left\{\left(I_{i}, t_{i}\right)\right\}_{i=1}^{n}$ of non-overlapping closed sub-intervals of $[a, b]$ and real numbers is called a $\delta$-fine partition of $[a, b]$ if $\left\{I_{i}\right\}_{i=1}^{n}$ is a partition of $[a, b]$ and $t_{i} \in I_{i} \subseteq\left(t_{i}-\delta\left(t_{i}\right), t_{i}+\delta\left(t_{i}\right)\right)$ for each $i \in\{1,2, \ldots, n\}$.

In what follows, $X$ is a locally convex topological vector space.

Definition 1. [8] A function $f:[a, b] \rightarrow X$ is Henstock integrable or HK-integrable on $[a, b]$, if there is an $\alpha \in X$ such that for any $\theta$-nbd $U$ there is a gauge $\delta$ on $[a, b]$ such that for any $\delta$-fine partition $P=\left\{\left(\left[x_{i-1}, x_{i}\right], t_{i}\right): 1 \leq i \leq n\right\}$ of $[a, b]$, we have

$$
\sum_{i=1}^{n}\left(x_{i}-x_{i-1}\right) f\left(t_{i}\right)-\alpha \in U \text {. }
$$

In this case, we write $f \in \mathcal{H}([a, b], X)$ and $(\mathcal{H}) \int_{a}^{b} f=\alpha$.

Definition 2. [9] A function $f:[a, b] \rightarrow X$ is strongly Henstock integrable or SH-integrable on $[a, b]$ if there is a function $F:[a, b] \rightarrow X$, called the primitive of $f$, and for every $\theta$-nbd $U$, there exists a gauge $\delta$ such that for any $\delta$-fine partition $D=\left\{\left(\left[u_{i}, v_{i}\right], t_{i}\right): 1 \leq i \leq n\right\}$, there exist $\theta$-nbds $U_{1}, U_{2}, \ldots, U_{n}$ such that $\sum_{i=1}^{n} U_{i} \subseteq U$ and $F\left(v_{i}\right)-F\left(u_{i}\right)-f\left(t_{i}\right)\left(v_{i}-u_{i}\right) \in$ $U_{i}$ for each $i \in\{1,2, \ldots, n\}$. In this case, we may write $f \in S H([a, b], X)$. The difference $F(b)-F(a)$ is the $S H$-integral of $f$ on $[a, b]$. In symbols, we write

$$
(S H) \int_{a}^{b} f=F(b)-F(a) .
$$

From the above definitions, it is easy to show that every $S H$ integrable function is $H K$ integrable.

Definition 3. [7] A function $F:[a, b] \rightarrow X$ is said to be absolutely continuous on $[a, b]$ (or $F$ is $A C$ on $[a, b]$ ) if for every $\theta$-nbd $U$, there exists an $\eta>0$ such that for any partial partition $D=\left\{\left[u_{i}, v_{i}\right]: 1 \leq i \leq n\right\}$ of $[a, b]$ with $\sum_{i=1}^{n}\left(v_{i}-u_{i}\right)<\eta$, there exist $\theta$-nbds $U_{1}, U_{2}, \ldots, U_{n}$ such that $\sum_{i=1}^{n} U_{i} \subseteq U$ and $F\left(v_{i}\right)-F\left(u_{i}\right) \in U_{i}$ for each $i \in\{1,2, \ldots, n\}$.

Definition 4. function $F:[a, b] \rightarrow X$ is said to be $A C^{*}(E)$, where $E \subseteq[a, b]$, if for every $\theta$-nbd $U$, there exists an $\eta>0$ such that for any partial partition $D=\left\{\left[u_{i}, v_{i}\right]: 1 \leq i \leq n\right\}$ of $[a, b]$ with $u_{i}$ or $v_{i} \in E$ and $\sum_{i=1}^{n}\left(v_{i}-u_{i}\right)<\eta$, there exist $\theta$-nbds $U_{1}, U_{2}, \ldots, U_{n}$ such that $\sum_{i=1}^{n} U_{i} \subseteq U$ and $F\left(v_{i}\right)-F\left(u_{i}\right) \in U_{i}$ for each $i \in\{1,2, \ldots, n\}$.

Definition 5. A function $F:[a, b] \rightarrow X$ is said to be $A C G^{*}$ on $[a, b]$ if there exists a collection $\left\{E_{i}\right\}_{i=1}^{\infty}$ of subsets of $[a, b]$ with $[a, b]=\bigcup_{i=1}^{\infty} E_{i}$ such that $F$ is $A C^{*}\left(E_{i}\right)$ for each $i \in \mathbb{N}$.

Definition 6. [9] Let $F:[a, b] \rightarrow X$ be a function and let $t \in[a, b]$. Then $F$ is differentiable at $t\left(F^{\prime}(t)\right.$ is the derivative of $F$ at $\left.t\right)$ if for every $\theta$-nbd $U$, there is a $\delta>0$ for which $F(v)-F(u)-F^{\prime}(t)(v-u) \in(v-u) U$ whenever $t \in[u, v] \subseteq[a, b]$ and $|v-u|<\delta$. 
Definition 7. A function $f:[a, b] \rightarrow X$ is said to be Denjoy integrable ( $\mathcal{D}^{*}$-integrable) on $[a, b]$ if there is a function $F:[a, b] \rightarrow X$, called the Denjoy primitive of $f$, which is $A C G^{*}$ on $[a, b]$ and $F^{\prime}(t)=f(t)$ almost everywhere on $[a, b]$. In this case, $F(b)-F(a)$ is the Denjoy integral of $f$ on $[a, b]$ and write

$$
\left(\mathcal{D}^{*}\right) \int_{a}^{b} f=F(b)-F(a) .
$$

Definition 8. A function $f:[a, b] \rightarrow X$ is said to be weak Denjoy integrable (wD $\mathcal{D}^{*}$ integrable) on $[a, b]$ if there is a function $F:[a, b] \rightarrow X$, called the weak Denjoy primitive of $f$, which is $A C G^{*}$ on $[a, b]$ and $\Delta(U, F, f)$ has measure zero for all $\theta$-nbds $U$, where

$$
\begin{gathered}
\Delta(U, F, f)=\{t \in[a, b]: \forall \delta>0, \exists[u, v] \subseteq[a, b] \text { with } t \in[u, v] \text { and }|v-u|<\delta \\
\text { such that } F(v)-F(u)-f(t)(v-u) \notin(v-u) U\} .
\end{gathered}
$$

We denote the weak Denjoy integral of $f$ on $[a, b]$ by

$$
\left(w \mathcal{D}^{*}\right) \int_{a}^{b} f=F(b)-F(a) .
$$

For a Banach space $X$, the concepts of $A C^{*}$ and $A C G^{*}$ are defined in the following way.

Definition 9. $[1,12] A$ function $F:[a, b] \rightarrow X$ is said to be $A C^{*}(E)$, where $E \subset[a, b]$, if for every $\epsilon>0$, there exists a $\delta>0$ such that for any partial partition $D=\left\{\left[u_{i}, v_{i}\right]: 1 \leq\right.$ $i \leq n\}$ of $[a, b]$ with $u_{i}$ or $v_{i} \in E$ and $\sum_{i=1}^{n}\left(v_{i}-u_{i}\right)<\delta, \sum_{i=1}^{n}\left\|F\left(v_{i}\right)-F\left(u_{i}\right)\right\|<\epsilon$.

Definition 10. $[1,12]$ A function $F:[a, b] \rightarrow X$ is said to be $A C G^{*}$ on $[a, b]$ if there exists a collection $\left\{E_{i}\right\}_{i=1}^{\infty}$ of subsets of $[a, b]$ with $[a, b]=\bigcup_{i=1}^{\infty} E_{i}$ such that $F$ is $A C^{*}\left(E_{i}\right)$ for each $i \in \mathbb{N}$.

Definition 11. [12] A function $f:[a, b] \rightarrow X$ is said to be Denjoy-Bochner integrable $\left(\mathcal{D}_{*} \mathcal{B}\right.$-integrable) on $[a, b]$ if there is an $A C G^{*}$-function $F:[a, b] \rightarrow X$ such that $F^{\prime}(t)=$ $f(t)$ almost everywhere on $[a, b]$.

\section{Results}

Remark 1. The conditions $A C$ and $A C^{*}$ are equivalent when $E=[a, b]$. Every absolutely continuous function on $[a, b]$ is also $A C G^{*}$ on $[a, b]$.

Example 1. Any function of the form $L(t)=t \boldsymbol{u}+\boldsymbol{v}$ is $A C G^{*}$ on $[a, b]$ where $\boldsymbol{u}, \boldsymbol{v} \in X$ is absolutely continuous (so also $A C G^{*}$ on $[a, b]$ ). In particular, every constant function is $A C G^{*}$ on $[a, b]$.

To see this, let $V$ be a given $\theta$-nbd. Let $U \subseteq V$ be an absorbing, balanced and convex $\theta$-nbd. Then there exists $\eta>0$ such that $r \mathbf{u} \in U$ for all $r \in \mathbb{R}$ with $|r|<\eta$. Let 
$D=\left\{\left[x_{i}, y_{i}\right]: 1 \leq i \leq n\right\}$ be a partial partition of $[a, b]$ with $\sum_{i=1}^{n}\left(y_{i}-x_{i}\right)=\eta^{*}<\eta$. Then $\mathbf{u} \in \frac{1}{\eta^{*}} U$ and $\left(y_{i}-x_{i}\right) \mathbf{u} \in U_{i}=\frac{y_{i}-x_{i}}{\eta^{*}} U$ for each $i \in\{1,2, \ldots, n\}$. Thus,

$$
\begin{aligned}
L\left(y_{i}\right)-L\left(x_{i}\right) & =y_{i} \mathbf{u}+\mathbf{v}-\left(x_{i} \mathbf{u}+\mathbf{v}\right)=\left(y_{i}-x_{i}\right) \mathbf{u} \\
& \in \frac{y_{i}-x_{i}}{\eta^{*}} U=U_{i} \text { for all } i \in\{1,2, \cdots, n\} .
\end{aligned}
$$

Since $\sum_{i=1}^{n} \frac{y_{i}-x_{i}}{\eta^{*}}=1$ and $U$ is convex, $\sum_{i=1}^{n} \frac{y_{i}-x_{i}}{\eta^{*}} U=\sum_{i=1}^{n} U_{i} \subseteq U$. Therefore, $L$ is absolutely continuous on $[a, b]$. Using the above definition, the following result follows.

Theorem 1. Let $A, B \subseteq[a, b]$.

(i) If $A \subseteq B$ and $F:[a, b] \rightarrow X$ is $A C^{*}(B)$, then $F$ is $A C^{*}(A)$.

(ii) If $F:[a, b] \rightarrow X$ is $A C^{*}(A)$ and $c \in \mathbb{R}$, then $c F$ is $A C^{*}(A)$.

(iii) If $G, H:[a, b] \rightarrow X$ are $A C^{*}(A)$, then $G+H$ is $A C^{*}(A)$.

(iv) If $F:[a, b] \rightarrow X$ is both $A C^{*}(A)$ and $A C^{*}(B)$, then $F$ is $A C^{*}(A \cup B)$.

Proof.

(i) This is immediate from Definition 4 .

(ii) The case $c=0$ is clear. Suppose $c \neq 0$. For any $\theta$-nbd $U$, there exists $\eta>0$ such that for any partial partition $D=\left\{\left[u_{i}, v_{i}\right]: 1 \leq i \leq n\right\}$ of $[a, b]$ with $u_{i}$ or $v_{i}$ in $A$ and (D) $\sum_{i=1}^{n}\left(v_{i}-u_{i}\right)<\eta$, there exist $\theta$-nbds $V_{1}, V_{2}, \ldots, V_{n}$ with $\sum V_{i} \subseteq \frac{1}{c} U$ for which $F\left(v_{i}\right)-F\left(u_{i}\right) \in V_{i}$ for $1 \leq i \leq n$. Set $U_{i}=c V_{i}$ for each $i \in\{1,2, \cdots, n\}$. Then $U_{i}$ is a $\theta$-nbd for $1 \leq i \leq n$ such that $\sum_{i=1}^{n} U_{i}=\sum_{i=1}^{n} c V_{i} \subseteq U$ and $c F\left(v_{i}\right)-c F\left(u_{i}\right) \in U_{i}$ for $1 \leq i \leq n$. Thus, $c F$ is $A C^{*}(A)$.

(iii) Let $U$ be a $\theta$-nbd $U$ and let $V$ and $W$ be $\theta$-nbds such that $V+W \subseteq U$. Since $G$ is $A C^{*}(A)$, there exists $\eta_{1}>0$ such that for any given a partial partition $\left\{\left[u_{i}, v_{i}\right]\right.$ : $1 \leq i \leq m\}$ of $[a, b]$ with $u_{i}$ or $v_{i}$ in $A$ and $\sum_{i=1}^{m}\left(v_{i}-u_{i}\right)<\eta_{1}$, there exist $\theta$-nbds $V_{1}, V_{2}, \ldots, V_{m}$ such that $\sum_{i=1}^{m} V_{i} \subseteq V$ and $G\left(v_{i}\right)-G\left(u_{i}\right) \in V_{i}$ for $1 \leq i \leq n$. Similarly, there exists $\eta_{2}>0$ such that for any partial partition $\left\{\left[u_{i}^{\prime}, v_{i}^{\prime}\right]: 1 \leq i \leq n\right\}$ of $[a, b]$ with $u_{i}^{\prime}$ or $v_{i}^{\prime}$ in $A$ and $\sum_{i=1}^{n}\left(v_{i}^{\prime}-u_{i}^{\prime}\right)<\eta_{2}$, there exist $\theta$-nbds $W_{1}, W_{2}, \ldots, W_{n}$ such that $\sum_{i=1}^{m} W_{i} \subseteq W$ and $H\left(v_{i}\right)-H\left(u_{i}\right) \in W_{i}$ for $1 \leq i \leq n$. Let $\eta=\min \left\{\eta_{1}, \eta_{2}\right\}$. Suppose $\left\{\left[x_{i}, y_{i}\right]: 1 \leq i \leq k\right\}$ is a partial partition of $[a, b]$ with $x_{i}$ or $y_{i}$ in $A$ such that $\sum_{i=1}^{k}\left(y_{i}-x_{i}\right)<\eta$. Since $\eta \leq \eta_{1}$ and $\eta \leq \eta_{2}$, there exist collections $\left\{V_{i}^{\prime}\right\}_{i=1}^{k}$ and $\left\{W_{i}^{\prime}\right\}_{i=1}^{k}$ of $\theta$-nbds such that $\sum_{i=1}^{k} V_{i}^{\prime} \subseteq V, \sum_{i=1}^{k} W_{i}^{\prime} \subseteq W$ and $G\left(y_{i}\right)-G\left(x_{i}\right) \in$ $V_{i}^{\prime}, H\left(y_{i}\right)-H\left(x_{i}\right) \in W_{i}^{\prime}$ for $1 \leq i \leq n$. Then $\sum_{i=1}^{k}\left(V_{i}+W_{i}\right) \subseteq V+W \subseteq U$ and for each $i \in\{1,2, \cdots, n\}$,

$$
F\left(v_{i}\right)+G\left(v_{i}\right)-\left(F\left(u_{i}\right)+G\left(u_{i}\right)\right)=F\left(v_{i}\right)-F\left(u_{i}\right)+G\left(v_{i}\right)-G\left(u_{i}\right) \in V_{i}+W_{i} .
$$

Therefore, $F+G$ is $A C^{*}(A)$. 
(iv) Let $U$ be a $\theta$-nbd and let $V$ and $W$ be $\theta$-nbds with $V+W \subseteq U$. Since $F$ is $A C^{*}(A)$, there exists $\eta_{1}>0$ such that for any partial partition $\left\{\left[u_{i}, v_{i}\right]: 1 \leq i \leq m\right\}$ with $u_{i}$ or $v_{i}$ in $A$ and $\sum_{i=1}^{m}\left(v_{i}-u_{i}\right)<\eta_{1}$, there exist $\theta$-nbds $V_{1}, V_{2}, \ldots, V_{m}$ with $\sum_{i=1}^{m} V_{i} \subseteq V$ such that $F\left(v_{i}\right)-F\left(u_{i}\right) \in V_{i}$ for $1 \leq i \leq n$. Likewise, because $F$ is $A C^{*}(B)$, there exists $\eta_{2}>0$ such that for any partial partition $\left\{\left[u_{i}^{\prime}, v_{i}^{\prime}\right]: 1 \leq i \leq n\right\}$ with $u_{i}^{\prime}$ or $v_{i}^{\prime}$ in $A$ and $\sum_{i=1}^{n}\left(v_{i}^{\prime}-u_{i}^{\prime}\right)<\eta_{2}$, there exist $\theta$-nbds $W_{1}, W_{2}, \ldots, W_{n}$ with $\sum_{i=1}^{n} W_{i} \subseteq W$ such that $F\left(y_{i}\right)-F\left(x_{i}\right) \in W_{i}$ for $1 \leq i \leq n$. Let $\eta=\min \left\{\eta_{1}, \eta_{2}\right\}$. Suppose $D=\left\{\left[x_{i}, y_{i}\right]: 1 \leq i \leq k\right\}$ is a partial partition of $[a, b]$ with $x_{i}$ or $y_{i} \in$ $A \cup B$ and $\sum_{i=1}^{k}\left(v_{i}-u_{i}\right)<\eta$. Let $D_{1}=\left\{\left[x_{i}, y_{i}\right] \in D: x_{i}\right.$ or $\left.y_{i} \in A\right\}$ and $D_{2}=\left\{\left[x_{i}, y_{i}\right] \in D \backslash D_{1}: x_{i}\right.$ or $\left.y_{i} \in B\right\}$. If $D_{1}=\varnothing$ or $D_{2}=\varnothing$, then we are done. So suppose that $D_{1} \neq \varnothing$ and $D_{2} \neq \varnothing$. By relabeling the elements of $D_{1}$ and $D_{2}$, we may write $D_{1}=\left\{\left[a_{i}, b_{i}\right]: 1 \leq i \leq k_{1}\right\}$ and $D_{2}=\left\{\left[a_{j}^{\prime}, b_{j}^{\prime}\right]: 1 \leq j \leq k_{2}\right\}$ where $k=k_{1}+k_{2}$. Then by assumption, there exist collections $\left\{V_{i}\right\}_{i=1}^{k_{1}}$ and $\left\{W_{i}\right\}_{i=1}^{k_{2}}$ of $\theta$ nbds such that $\sum_{i=1}^{k_{1}} V_{i} \subseteq V$ and $\sum_{i=1}^{k_{2}} W_{i} \subseteq W$ for which $F\left(b_{i}\right)-F\left(a_{i}\right) \in V_{i}$ for each $i \in\left\{1,2, \cdots, k_{1}\right\}$ and $F\left(b_{i}^{\prime}\right)-F\left(a_{i}^{\prime}\right) \in W_{i}$ for each $i \in\left\{1,2, \cdots, k_{2}\right\}$. Let $V_{k_{1}+j}=W_{j}$ for each $j \in\left\{1,2, \ldots, k_{2}\right\}$. Then $V_{1}, V_{2}, \ldots, V_{k_{1}}, V_{k_{1}+1}, \ldots, V_{k_{1}+k_{2}-1}, V_{k}$ are $\theta$-nbds and $\sum_{i=1}^{k} V_{i}=\sum_{i=1}^{k_{1}} V_{i}+\sum_{i=1}^{k_{2}} W_{i} \subseteq V+W \subseteq U$. Therefore, $F$ is $A C^{*}(A \cup B)$.

Remark 2. If a function $F$ is $A C G^{*}$ on $[a, b]$, then $[a, b]$ is the union of sets in some collection $\left\{Y_{i}\right\}_{i=1}^{\infty}$ of subsets of $[a, b]$ for which $F$ is $A C^{*}\left(Y_{i}\right)$ for each $i \in \mathbb{N}$. We may assume that the sets are disjoint. In fact, the collection $\left\{Z_{i}\right\}_{i=1}^{\infty}$ is mutually disjoint and satisfies the condition for $A C G^{*}$ where $Z_{i}=Y_{i} \backslash\left(Y_{1} \cup Y_{2} \cup \cdots \cup Y_{i-1}\right) \subseteq Y_{i}$.

The next result follows from (ii)and (iii) of Theorem 1.

Theorem 2. Let $F, G:[a, b] \rightarrow X$ be $A C G^{*}$ on $[a, b]$ and let $c \in \mathbb{R}$. Then $c F$ and $F+G$ are $A C G^{*}$ on $[a, b]$.

Theorem 3. If $F:[a, b] \rightarrow X$ is an $A C G^{*}$ function, then $F$ is continuous.

Proof. Let $U$ be an open set in $X$. Let $c \in F^{-1}(U)$. Since $F$ is $A C G^{*}$, there exists a countable collection $\left\{E_{i}\right\}_{i=1}^{\infty}$ of subsets of $[a, b]$ whose union is $[a, b]$ such that $F$ is $A C^{*}\left(E_{i}\right)$ for each $i \in \mathbb{N}$. Let $c \in E_{k}$ for some $k \in \mathbb{N}$. Clearly, $U-F(c)$ is a $\theta$-nbd. Let $W$ be a balanced $\theta$-nbd such that $W \subseteq U-F(c)$ and let $\eta$ be a positive number associated with $F, W, E_{k}$ according to the definition of $A C^{*}$. Let $x \in[a, b]$ be such that $|x-c|<\eta$ and $x \neq c$. Then $D=\{[x, c]\}$ or $D=\{[c, x]\}$ is a partial partition of $[a, b]$ depending on whether $c>x$ or $c<x$. By assumption, there exists a $\theta$-nbd $V$ with $V \subseteq W$ such that $F(c)-F(x) \in V$ or $F(x)-F(c) \in V$. Since $W$ is balanced, $F(x)-F(c) \in W \subseteq U-F(c)$. Hence, $x \in F^{-1}(U)$, implying that $(c-\eta, c+\eta) \subseteq F^{-1}(U)$. Therefore, $F$ is continuous on $[a, b]$.

The next two results can also be proved using the definitions. 
Theorem 4. Let $A \subseteq[c, d] \subseteq[a, b]$ and $F:[a, b] \rightarrow X$ be $A C^{*}(A)$. Then the restriction $\left.F\right|_{[c, d]}$ of $F$ to $[c, d]$ is $A C^{*}(A)$. In particular, if $F$ is $A C G^{*}$ on $[a, b]$, then $\left.F\right|_{[c, d]}$ is $A C G^{*}$ on $[c, d]$.

Theorem 5. Let F, G, $f, g:[a, b] \rightarrow X$ be functions. Then each of following holds:

(i) $\left\{t \in[a, b]: F^{\prime}(t) \neq f(t)\right\}=\bigcup_{\theta-n b d U} \Delta(U, F, f)$.

(ii) $\Delta(U, c F, c f)=\Delta\left(\frac{1}{c} U, F, f\right)$ for each $\theta$-nbd $U$ and $0 \neq c \in \mathbb{R}$.

(iii) $\Delta(U, F+G, f+g) \subseteq \Delta\left(\frac{1}{2} U, F, f\right) \cup \Delta\left(\frac{1}{2} U, G, g\right)$ for each convex $\theta$-nbd $U$.

Theorem 6. Let $F:[a, b] \rightarrow X$ be $A C G^{*}$ on $[a, b]$ and let $f:[a, b] \rightarrow X$ be the zero function. If $\Delta(U, F, f)$ is of measure zero for all $\theta$-nbds $U$, then $F$ is a constant function.

Proof. Let $a<c \leq b$. Since $F$ is $A C G^{*}$ on $[a, b], F \mid[a, c]$ is $A C G^{*}$ on $[a, c]$. This implies that there is a disjoint countable collection $\left\{Y_{i}\right\}_{i=1}^{\infty}$ of subsets of $[a, c]$ such that $[a, c]=\bigcup_{i=1}^{\infty} Y_{i}$ and $F$ is $A C^{*}\left(Y_{i}\right)$ for each $i \in \mathbb{N}$. Let $U$ be a given $\theta$-nbd. Then there exist an absorbing, balanced and convex $\theta$-nbd $V$ such that $(c-a+1) V \subseteq U$. Note that if $t \in[a, c] \backslash \Delta\left(V,\left.F\right|_{[a, c]},\left.f\right|_{[a, c]}\right)$, then there exists $\delta_{0}(t)>0$ such that $F(v)-F(u) \in(v-u) V$ whenever $t \in[u, v] \subseteq[a, c]$ and $|v-u|<\delta_{0}(t)$. Let $A_{i}=\Delta\left(V,\left.F\right|_{[a, c]},\left.f\right|_{[a, c]}\right) \cap Y_{i}$ for each $i \in \mathbb{N}$. Then $\left.F\right|_{[a, c]}$ is $A C^{*}\left(A_{i}\right)$ for each $i \in \mathbb{N}$. Thus, for each $i \in \mathbb{N}$, there exist $\eta_{i}>0$ such that for every partial partition $D=\left\{\left[u_{j}, v_{j}\right]: 1 \leq j \leq n\right\}$ of $[a, c]$ with $u_{j}$ or $v_{j}$ in $A_{i}$ and $\sum_{j=1}^{n}\left(v_{j}-u_{j}\right)<\eta_{i}$, there exist $\theta$-nbds $V_{i, 1}, V_{i, 2}, \ldots, V_{i, n_{i}}$ with $\sum_{j=1}^{n_{i}} V_{i, j} \subseteq \frac{1}{2^{i}} V$ and $F\left(v_{j}\right)-F\left(u_{j}\right) \in V_{i, j}$ for all $1 \leq j \leq n_{i}$. Now, by the definition of $A_{i}, m\left(A_{i}\right)=0$ for all $i \in \mathbb{N}$ where $m$ is the Lebesgue measure. Hence, there exists a collection $\left\{G_{i}\right\}_{i=1}^{\infty}$ of open sets with $A_{i} \subseteq G_{i}$ and $m\left(G_{i}\right)<\eta_{i}$ for each $i \in \mathbb{N}$. Thus, for every $t \in A_{i}$ there is a real number $\delta_{i}(t)$ such that $\left(t-\delta_{i}(t), t+\delta_{i}(t)\right) \subseteq G_{i}$. Define $\delta(t)=\delta_{0}(t)$ if $t \in[a, c] \backslash \Delta(V, F, f)$ and $\delta(t)=\delta_{i}(t)$ if $t \in A_{i}$ for some $i \geq 1$. Let $D=\left\{\left(\left[x_{j}, y_{j}\right], t_{j}\right): 1 \leq j \leq k\right\}$ be a $\delta$-fine partition of $[a, b]$. Then the set of intervals in $D$ is a disjoint union of $D_{1}$ and $D_{2}$ where $D_{1}=\left\{\left[x_{j}, y_{j}\right]:\left(\left[x_{j}, y_{j}\right], t_{j}\right) \in D\right.$ and $\left.t_{j} \in[a, c] \backslash \Delta\left(V,\left.F\right|_{[a, c]},\left.f\right|_{[a, c]}\right)\right\}$ and $D_{2}=\left\{\left[x_{j}, y_{j}\right]:\left(\left[x_{j}, y_{j}\right], t_{j}\right) \in D\right.$ and $\left.t_{j} \in \Delta\left(V,\left.F\right|_{[a, c]},\left.f\right|_{[a, c]}\right)\right\}$. If $\left[x_{i}, y_{i}\right] \in D_{1}$, then $\left[x_{i}, y_{i}\right] \subseteq\left(t_{i}-\delta\left(t_{i}\right), t_{i}+\delta\left(t_{i}\right)\right)$ because $D$ is $\delta$-fine. Since $t_{i} \in[a, c] \backslash \Delta\left(V,\left.F\right|_{[a, c]},\left.f\right|_{[a, c]}\right)$, $F\left(y_{i}\right)-F\left(x_{i}\right) \in\left(y_{i}-x_{i}\right) V$. If $\left[x_{i}, y_{i}\right] \in D_{2}$, then $t_{i} \in A_{j}$ for exactly one $j \geq 1$ with $\left[x_{i}, y_{i}\right] \subseteq\left(t_{i}-\delta\left(t_{i}\right), t_{i}+\delta\left(t_{i}\right)\right)=\left(t_{i}-\delta_{j}\left(t_{i}\right), t_{i}+\delta_{j}\left(t_{i}\right)\right) \subseteq G_{j}$. Here, we may assume that $x_{i} \in A_{j}$ or $y_{i} \in A_{j}$ (otherwise, we replace $\left[x_{i}, y_{i}\right]$ with the intervals $\left[x_{i}, t_{i}\right]$ and $\left.\left[t_{i}, y_{i}\right]\right)$. Hence, the union of the non-overlapping intervals $\left[x_{i}, y_{i}\right]$ with $t_{i} \in A_{j}$ is contained in $G_{j}$. This implies that $\sum_{t_{i} \in A_{j}}\left(y_{i}-x_{i}\right) \leq m^{*}\left(G_{j}\right)<\eta_{j}$. Thus, there exist $\theta$-nbds $V_{j, 1}, V_{j, 2}, \ldots, V_{j, n_{j}}$ with $\sum_{i=1}^{n_{j}} V_{j, i} \subseteq \frac{1}{2^{j}} V$ and $F\left(y_{i}\right)-F\left(x_{i}\right) \in V_{j, i}$ for each $i \in\left\{1,2, \ldots, n_{j}\right\}$. Let $K=\left\{i \in\{1,2, \ldots, k\}: t_{i} \in[a, c] \backslash \Delta\left(V,\left.F\right|_{[a, c]},\left.f\right|_{[a, c]}\right)\right\}$ and $S=\left\{j \in \mathbb{N}: t_{i} \in\right.$ $A_{j}$ for some $\left.i \in\{1,2, \ldots, k\}\right\}$. Consequently, by convexity of $V$,

$$
\begin{aligned}
F(c)-F(a) & =\sum_{\left[x_{i}, y_{i}\right] \in D_{1}}\left(F\left(y_{i}\right)-F\left(x_{i}\right)\right)+\sum_{\left[x_{i}, y_{i}\right] \in D_{2}}\left(F\left(y_{i}\right)-F\left(x_{i}\right)\right) \\
& \in \sum_{i \in K}\left(y_{i}-x_{i}\right) V+\sum_{j \in S} \frac{1}{2^{j}} V
\end{aligned}
$$




$$
\subseteq(c-a) V+V \subseteq(c-a+1) V \subseteq U
$$

Since $U$ was arbitrarily chosen, $F(a)=F(c)$. Therefore, $F$ is a constant function.

\section{The Denjoy and Weak Denjoy Integrals}

Theorem 7. Let $f:[a, b] \rightarrow X$ be weak Denjoy integrable on $[a, b]$. Then the weak Denjoy integral of $f$ is unique.

Proof. Let $F_{1}$ and $F_{2}$ be weak Denjoy primitives of $f$. Since $F_{1}$ and $F_{2}$ are $A C G^{*}$ on $[a, b], F_{1}-F_{2}$ is $A C G^{*}$ on $[a, b]$ by Theorem 2. Let $U$ be a $\theta$-nbd and let $V$ be a balanced $\theta$ nbds with $V+V \subseteq U$. Then both $\Delta\left(V, F_{1}, f\right)$ and $\Delta\left(V, F_{2}, f\right)$ have measure zero. Let $t \in \Delta\left(U, F_{1}-F_{2}, 0\right)$ where 0 is the zero function on $[a, b]$. Suppose $t \notin \Delta\left(V, F_{1}, f\right) \cup$ $\Delta\left(V, F_{2}, f\right)$. Then there exists $\delta>0$ such that $F_{1}(v)-F_{1}(u)-f(t)(v-u) \in(v-u) V$ and $F_{2}(v)-F_{2}(u)-f(t)(v-u) \in(v-u) V=-(v-u) V$ (since $V$ is balanced) whenever $t \in[u, v] \subseteq[a, b]$ and $|v-u|<\delta$. It follows that

$$
\begin{aligned}
& F_{1}(v)-F_{1}(u)-\left(F_{2}(v)-F_{2}(u)\right)= F_{1}(v)-F_{1}(u)-f(t)(v-u) \\
&-\left(F_{2}(v)-F_{2}(u)-f(t)(v-u)\right) \\
& \in(v-u) V+(v-u) V \subseteq(v-u) U .
\end{aligned}
$$

This implies that $t \notin \Delta\left(U, F_{1}-F_{2}, 0\right)$, contrary to our assumption. Hence, $\Delta\left(U, F_{1}-\right.$ $\left.F_{2}, 0\right) \subseteq \Delta\left(V, F_{1}, f\right) \cup \Delta\left(V, F_{2}, f\right)$. Consequently, $\Delta\left(U, F_{1}-F_{2}, 0\right)$ has measure zero for all $\theta$-nbdss $U$. By Theorem 6 , there is $\alpha \in X$ such that $F_{1}-F_{2}=\alpha$ on $[a, b]$, that is, $F_{1}(b)-F_{1}(a)=F_{2}(b)+\alpha-\left(F_{2}(a)+\alpha\right)=F_{2}(b)-F_{2}(a)$. This proves the assertion.

Similarly, the Denjoy integral of a function, if it exists, is also unique. Further, it can easily be proved that Denjoy integrability implies weak Denjoy integrability.

Theorem 8. If $f:[a, b] \rightarrow X$ is Denjoy integrable on $[a, b]$, then its Denjoy integral is unique.

Theorem 9. If $f:[a, b] \rightarrow X$ is Denjoy integrable on $[a, b]$, then it is weak Denjoy integrable on $[a, b]$. Moreover, their primitives and integrals coincide.

Example 2. The constant function $f(t)=\alpha$ for all $t \in[a, b]$, where $\alpha \in X$, is Denjoy integrable on $[a, b]$ and

$$
\left(\mathcal{D}^{*}\right) \int_{a}^{b} f=(b-a) \alpha .
$$

Indeed, $F(t)=t \cdot \alpha$ for all $t \in[a, b]$ is absolutely continuous and $F^{\prime}(t)=\alpha=f(t)$ on $[a, b]$. Hence, by Remark $1, F$ is a primitive of $f$ and

$$
\left(\mathcal{D}^{*}\right) \int_{a}^{b} f=F(b)-F(a)=b \cdot \alpha-a \cdot \alpha=(b-a) \alpha .
$$


Theorem 10. Let $f, g:[a, b] \rightarrow X$ be weak Denjoy integrable functions and $c \in \mathbb{R}$. Then each of the following statements holds.

(i) cf is weak Denjoy integrable and

$$
\left(w \mathcal{D}^{*}\right) \int_{a}^{b}(c f)=c \cdot\left(w \mathcal{D}^{*}\right) \int_{a}^{b} f
$$

(ii) $f+g$ is weak Denjoy integrable and

$$
\left(w \mathcal{D}^{*}\right) \int_{a}^{b}(f+g)=\left(w \mathcal{D}^{*}\right) \int_{a}^{b} f+\left(w \mathcal{D}^{*}\right) \int_{a}^{b} g .
$$

Proof. Let $F$ and $G$ be weak Denjoy primitives of $f$ and $g$, respectively.

(i) By Theorem 2, $c F$ is $A C G^{*}$ on $[a, b]$. The result is clear if $c=0$. So suppose $c \neq 0$. Then $\Delta(U, c F, c f)=\Delta\left(\frac{1}{c} U, F, f\right)$ by Theorem $5(i i)$. Since $\Delta\left(\frac{1}{c} U, F, f\right)$ has measure zero for all $\theta$-nbds $U, \Delta(U, c F, c f)$ has measure zero for all $\theta$-nbds $U$. Hence, $c f$ is weak Denjoy integrable with primitive $c F$ on $[a, b]$. Furthermore,

$$
\left(w \mathcal{D}^{*}\right) \int_{a}^{b}(c f)=c F(b)-c F(a)=c \cdot\left(w \mathcal{D}^{*}\right) \int_{a}^{b} f .
$$

(ii) The function $F+G$ is $A C G^{*}$ on $[a, b]$ by Theorem 2. Let $U$ be a given $\theta$-nbd and let $V \subseteq U$ be a convex $\theta$-nbd. Then $\Delta(V, F+G, f+g) \subseteq \Delta\left(\frac{1}{2} V, F, f\right) \cup \Delta\left(\frac{1}{2} V, G, g\right)$ by Theorem $5($ iii $)$. Since both $\Delta\left(\frac{1}{2} V, F, f\right)$ and $\Delta\left(\frac{1}{2} V, G, g\right)$ have measure zero, $\Delta(V, F+G, f+g)$ is of measure zero. Thus, $\Delta(U, F+G, f+g)$ has measure zero, implying that $f+g$ is weak Denjoy integrable with primitive $F+G$ on $[a, b]$ and

$$
\left(w \mathcal{D}^{*}\right) \int_{a}^{b}(f+g)=(F+G)(b)-(F+G)(a)=\left(w \mathcal{D}^{*}\right) \int_{a}^{b} f+\left(w \mathcal{D}^{*}\right) \int_{a}^{b} f .
$$

Remark 3. An analog of Theorem 10 holds for the Denjoy integral and the proof is easy.

For the next result, one may also refer to [6].

Theorem 11. Let $f:[a, b] \rightarrow X$. If $f=\theta$ almost everywhere, then $f$ is $S H$-integrable and $(S H) \int_{a}^{b} f=\theta$.

Proof. We show that $F:[a, b] \rightarrow X$ defined by $F(t)=\theta$ is an $S H$ primitive of $f$. Let $V$ be a $\theta$-nbd. Let $U$ be an absorbing, balanced, and convex $\theta$-nbd with $U \subseteq V$. Let $S=\{t \in[a, b]: f(t) \neq \theta\}$ and $E_{k}=\{t \in S: f(t) \in k U \backslash(k-1) U\}$ for each positive integer $k$. Then the collection $\left\{E_{i}\right\}_{i=1}^{\infty}$ is pairwise disjoint. Let $t \in S$. Then $f(t) \neq \theta$. Since $U$ is absorbing, there is a positive integer $r$ such that $f(t) \in r U$. We may choose $r$ to be the smallest positive integer with this property. Thus, $f(t) \in E_{r}$, showing that $S \subseteq \bigcup_{i=1}^{\infty} E_{i}$. Since $\bigcup_{i=1}^{\infty} E_{i} \subseteq S, S=\bigcup_{i=1}^{\infty} E_{i}$. Also, $m(S)=0$ implies that $m\left(E_{k}\right)=0$ 
for each positive integer $k$. Thus, for each positive integer $k$, there exists an open set $G_{k}$ such that $E_{k} \subseteq G_{k}$ and $m\left(G_{k}\right)<\frac{1}{k 2^{k}}$. Set $\delta(t)=1$ if $t \in[a, b] \backslash S$ and let $\delta(t)>0$ be a real number such that $(t-\delta(t), t+\delta(t)) \subseteq G_{k}$, if $t \in E_{k}$. Let $D=\left\{\left(\left[u_{i}, v_{i}\right], t_{i}\right): 1 \leq i \leq n\right\}$ be a $\delta$-fine partition of $[a, b]$. Let $D_{0}$ be the elements in $D$ for which the tags are not in $S$ and let $D_{k}=\left\{\left(\left[u_{i}, v_{i}\right], t_{i}\right) \in D: t_{i} \in E_{k}\right\}$ for each positive integer $k$. Then $\Phi_{U}(f(t)) \leq j$ and $\left[u_{i}, v_{i}\right] \subseteq\left(t_{i}-\delta\left(t_{i}\right), t_{i}+\delta\left(t_{i}\right)\right) \subseteq G_{j}$ for each $t \in E_{j}$. Hence, $\bigcup\left\{\left[u_{i}, v_{i}\right]: t_{i} \in E_{j}\right\} \subseteq G_{j}$. So, $\sum_{t_{i} \in E_{j}}\left(v_{i}-u_{i}\right) \leq m\left(G_{j}\right)<\frac{1}{j 2^{j}}$. Consequently,

$$
\begin{aligned}
(D) \sum \Phi_{V}(-f(t)(v-u)) \leq & (D) \sum \Phi_{U}(-f(t)(v-u)) \\
= & (D) \sum \Phi_{U}(-f(t)(v-u)) \\
& \quad+\left(D \backslash D_{0}\right) \sum \Phi_{U}\left(-f\left(t_{i}\right)\left(v_{i}-u_{i}\right)\right) \\
= & \left(D \backslash D_{0}\right) \sum \Phi_{U}(-f(t)(v-u)) \\
\leq & \sum_{j=1}^{\infty} \sum_{t_{i} \in E_{j}}\left(v_{i}-u_{i}\right) \Phi_{U}\left(-f\left(t_{i}\right)\right) \\
\leq & \sum_{j=1}^{\infty} \sum_{t_{i} \in E_{j}}\left(v_{i}-u_{i}\right) j \\
& <\sum_{j=1}^{\infty} \frac{1}{j 2^{j}} j=1
\end{aligned}
$$

Let $\epsilon=1-(D) \sum \Phi_{U}(-f(t)(v-u))>0$. For each $i \in\{1,2, \ldots, n\}$, let $r_{i}=\Phi_{U}\left(-f\left(t_{i}\right)\left(v_{i}-\right.\right.$ $\left.\left.u_{i}\right)\right)+\frac{\epsilon}{n}$. Because $U$ is balanced,

$$
\begin{aligned}
F\left(v_{i}\right)-F\left(u_{i}\right)-f\left(t_{i}\right)\left(v_{i}-u_{i}\right) & =\theta-\theta-f\left(t_{i}\right)\left(v_{i}-u_{i}\right) \\
& =-f\left(t_{i}\right)\left(v_{i}-u_{i}\right) \in r_{i} U .
\end{aligned}
$$

Note that $\sum_{i=1}^{n} r_{i}=\sum_{i=1}^{n}\left(\Phi_{U}\left(-f\left(t_{i}\right)\left(v_{i}-u_{i}\right)\right)+\frac{\epsilon}{n}\right)=1$. Since $U$ is convex, $\sum_{i=1}^{n}\left(r_{i} U\right) \subseteq$ $U$. Thus, $f$ is $S H$-integrable and $(S H) \int_{a}^{b} f=F(b)-F(a)=\theta-\theta=\theta$.

Theorem 12. If $f:[a, b] \rightarrow X$ is weak Denjoy integrable on $[a, b]$, then it is $S H$ integrable on $[a, b]$.

Proof. Let $F:[a, b] \rightarrow X$ be a weak Denjoy primitive of $f$. Let $U$ be a $\theta$-nbd and let $V$ be an absorbing, balanced and convex $\theta$-nbd such that $(2+b-a) V \subseteq U$. Let $f_{0}=f \cdot 1_{\Delta(V, F, f)}$. Since $f$ weak Denjoy integrable on $[a, b], \Delta(V, F, f)$ is of measure zero. Hence, $f_{0}(t)=\theta$ almost everywhere on $[a, b]$. By Theorem 11, there is a gauge $\delta_{0}$ such that for every $\delta_{0}$-fine partition $D=\left\{\left(\left[u_{i}, v_{i}\right], t_{i}\right): 1 \leq i \leq n\right\}$ of $[a, b]$, there exist $\theta$-nbds $U_{1}, U_{2}, \ldots, U_{n}$ with $\sum_{i=1}^{n} U_{i} \subseteq V$ and $-f_{0}\left(t_{i}\right)\left(v_{i}-u_{i}\right) \in U_{i}$. Now, since $F$ is $A C G^{*}$ on $[a, b]$, there is a disjoint collection $\left\{Y_{i}\right\}_{i=1}^{\infty}$ of subsets of $[a, b]$ with $[a, b]=\bigcup_{i=1}^{\infty} Y_{i}$ such that $F$ is $A C^{*}\left(Y_{i}\right)$ for all $i \in \mathbb{N}$. For each $i \in \mathbb{N}$, let $E_{i}=\Delta(U, F, f) \cap Y_{i}$. Then $F$ is 
$A C^{*}\left(E_{i}\right)$ and $E_{i}$ is of measure zero for all $i \in \mathbb{N}$. By definition, for each $i \in \mathbb{N}$, there is an $\eta_{i}>0$ such that for every partial partition $D=\left\{\left[u_{j}, v_{j}\right]: 1 \leq j \leq n\right\}$ of $[a, b]$ with $u_{j}$ or $v_{j} \in E_{i}$ and $\sum_{j=1}^{n}\left(v_{j}-u_{j}\right)<\eta_{i}$, there exist $\theta$-nbds $U_{1}, U_{2}, \ldots, U_{n}$ such that $\sum_{j=1}^{n} U_{j} \subseteq \frac{1}{2^{i}} V$ and $F\left(v_{j}\right)-F\left(u_{j}\right) \in U_{j}$ for $1 \leq j \leq n$. Also, since $m\left(E_{i}\right)=0$ for all $i \in \mathbb{N}$, there is a collection $\left\{G_{i}\right\}_{i=1}^{\infty}$ of open sets such that $E_{i} \subseteq G_{i}$ and $m\left(G_{i}\right)<\eta_{i}$ for all $i \in \mathbb{N}$. If $t \in \Delta(V, F, f)$, then $t \in E_{j} \subset G_{j}$ for some $j \in \mathbb{N}$. In this case, we choose $\delta_{1}(t)>0$ be such that $\left(t-\delta_{1}(t), t+\delta_{1}(t)\right) \subseteq G_{j}$. If $t \in[a, b] \backslash \Delta(V, F, f)$, then let $\delta_{2}(t)>0$ such that $F(v)-F(u)-f(t)(v-u) \in(v-u) V$ whenever $t \in[u, v] \subseteq\left(t-\delta_{2}(t), t+\delta_{2}(t)\right)$. Define $\delta$ as follows:

$$
\delta(t)=\left\{\begin{array}{ll}
\min \left\{\delta_{0}(t), \delta_{1}(t)\right\} & \text { if } t \in \Delta(V, F, f) \\
\delta_{2}(t) & \text { otherwise. }
\end{array} .\right.
$$

Let $D=\left\{\left(\left[u_{j}, v_{j}\right], t_{j}\right): 1 \leq j \leq n\right\}$ be a $\delta$-fine partition of $[a, b]$. Then $D=D_{1} \cup D_{2}$ where $D_{1}=\left\{\left(\left[u_{j}, v_{j}\right], t_{j}\right): t_{j} \in \Delta(V, F, f)\right\}$ and $D_{2}=\left\{\left(\left[u_{j}, v_{j}\right], t_{j}\right): t_{j} \in[a, b] \backslash \Delta(V, F, f)\right\}$. Let $S=\left\{i \in \mathbb{N}: t_{j} \in E_{i}\right.$ for some $\left.1 \leq j \leq n\right\}$. For each $i \in S$, let $D_{1, i}=\left\{\left(\left[u_{j}, v_{j}\right], t_{j}\right) \in\right.$ $\left.D_{1}: t_{j} \in E_{i}\right\}$. Then $D_{1}=\bigcup_{i \in S} D_{1, i}$. Since $D_{1}$ is a $\delta_{0}$-fine partial partition of $[a, b]$, there exist $\theta$-nbds $V_{i, j}$ such that $\sum_{i \in S}(D) \sum V_{i, j} \subseteq V$ and $-f\left(t_{j}\right)\left(v_{j}-u_{j}\right) \in V_{i, j}$ for $\left(\left[u_{j}, v_{j}\right], t_{j}\right) \in D_{1, i}$. Also, for each $i \in S$, we have $\bigcup_{t_{j} \in E_{i}}\left(u_{j}, v_{j}\right) \subseteq G_{i}$ implying that $\left(D_{1, i}\right) \sum\left(v_{j}-u_{j}\right)<\eta_{i}$. Hence, there are $\theta$-nbds $U_{i, j}$ such that $\left(D_{1, i}\right) \sum U_{i, j} \subseteq \frac{1}{2^{i}} V$ and $F\left(v_{j}\right)-F\left(u_{j}\right) \in U_{i, j}$ for $\left(\left[u_{j}, v_{j}\right], t_{j}\right) \in D_{1, i}$. Consequently, $F\left(v_{j}\right)-F\left(u_{j}\right)-f\left(t_{i}\right)\left(v_{j}-u_{j}\right) \in$ $U_{i, j}+V_{i, j}$ for $\left(\left[u_{j}, v_{j}\right], t_{j}\right) \in D_{1, i}$. Let $K=\left\{j \in\{1,2, \ldots, n\}: t_{j} \in[a, b] \backslash \Delta(V, F, f)\right\}$. Then $F\left(v_{j}\right)-F\left(u_{j}\right)-f\left(t_{j}\right)\left(v_{j}-u_{j}\right) \in\left(v_{j}-u_{j}\right) V$ for each $j \in K$ and $\sum_{j \in K}\left(v_{j}-u_{j}\right) V \subseteq(b-a) V$. Let $W_{i, j}=U_{i, j}+V_{i, j}$ for $\left(\left[u_{j}, v_{j}\right], t_{j}\right) \in D_{1, i}$ and $i \in S_{i}$ and $W_{j}=\left(v_{j}-u_{j}\right) V$ for $j \in K$. Then $F\left(v_{j}\right)-F\left(u_{j}\right)-f\left(t_{j}\right)\left(v_{j}-u_{j}\right) \in W_{j}$ for each $1 \leq j \leq n$ and

$$
\begin{aligned}
\sum_{i \in S}\left(D_{1, i}\right) \sum W_{i, j}+\sum_{j \in K} W_{j} & =\sum_{i \in S}\left(D_{1, i}\right) \sum\left(U_{i, j}+V_{i, j}\right)+\sum_{j \in K}\left(v_{j}-u_{j}\right) V \\
& \subseteq \sum_{i \in S} \frac{1}{2^{i}} V+\sum_{i \in S}\left(D_{1, i}\right) \sum V_{i, j}+(b-a) V \\
& \subseteq V+V+(b-a) V \subseteq(2+b-a) V \subseteq U .
\end{aligned}
$$

Therefore, $f$ is $S H$ integrable on $[a, b]$.

One difficulty that one may encounter in showing the converse (if it were true) of Theorem 12 is in dealing with the "differentiabilty" aspect. In the Banach-valued case, the proof in moving from the strong Henstock integral to $\mathcal{D}_{*} \mathcal{B}$ uses the condition of the Henstock Lemma (which the strong Henstock integral possesses) to prove differentiability. However, the $S H$-integral defined in this paper does not possess a similar property.

We now show that for functions taking values in a Banach space $X$, the Denjoy-Bochner integral defined by Solodov in [12] is stronger than the Denjoy integral.

Theorem 13. Let $X$ be a Banach space. If $f:[a, b] \rightarrow X$ is $\mathcal{D}_{*} \mathcal{B}$-integrable, then it is $\mathcal{D}^{*}$-integrable. 
Proof. Suppose that $f$ is $\mathcal{D}_{*} \mathcal{B}$-integrable on $[a, b]$. Let $F$ be an $A C G^{*}$-function such that $F^{\prime}(t)=f(t)$ a.e. on $[a, b]$. Let $\left\{E_{i}\right\}_{i=1}^{\infty}$ be a collection of subsets of $[a, b]$ with $[a, b]=\bigcup_{i=1}^{\infty} E_{i}$ such that $F$ is $A C^{*}\left(E_{i}\right)$ for each $i \in \mathbb{N}$. Let $U$ be a $\theta$-neighborhood. Then there exists $\epsilon>0$ such that $B_{\epsilon} \subseteq U$. Let $k \in \mathbb{N}$. Since $F$ is $A C^{*}\left(E_{k}\right)$, there exists a $\delta>0$ such that for any partial partition $D=\left\{\left(\left[u_{i}, v_{i}\right], t_{i}\right): 1 \leq i \leq n\right\}$ of $[a, b]$ with $u_{i} \in E_{k}$ or $v_{i} \in E_{k}$ and $\sum_{i=1}^{n}\left(v_{i}-u_{i}\right)<\delta$, we have $\sum_{i=1}^{n}\left\|F\left(v_{i}\right)-F\left(u_{i}\right)\right\|<\epsilon$. Choose positive numbers $\epsilon_{1}, \epsilon_{2}, \ldots, \epsilon_{n}$ such that $\left\|F\left(v_{i}\right)-F\left(u_{i}\right)\right\|<\epsilon_{i}$ for each $i \in\{1,2, \ldots, n\}$ and $\epsilon_{1}+\epsilon_{2}+\ldots+\epsilon_{n} \leq \epsilon$. Let $U_{i}=B_{\epsilon_{i}}=\left\{x \in X:\|x\|<\epsilon_{i}\right\}$ for each $i \in\{1,2, \ldots, n\}$. Then $\sum_{i=1}^{n} U_{i} \subseteq B_{\epsilon} \subseteq U$ and $F\left(v_{i}\right)-F\left(u_{i}\right) \in U_{i}$ for each $i \in\{1,2, \ldots, n\}$. Hence, $F$ is $A C^{*}\left(E_{k}\right)$ in the sense of Definition 4. Therefore, $F$ is an $A C G^{*}$-function in the sense of Definition 5. Next, let $E=\left\{t^{\prime} \in[a, b]: F^{\prime}(t)=f(t)\right\}$. Let $V$ be a $\theta$-neighborhood and let $\epsilon>0$ such that $B_{\epsilon} \subseteq V$. By assumption, there exists $\delta>0$ such that that $\left\|\frac{1}{v-u}\left[F(v)-F(u)-F^{\prime}(t)(v-u)\right]\right\|<\epsilon$ whenever $t \in[u, v] \cap E \subseteq[a, b] \cap E$ and $|v-u|<\delta$. This implies that $\frac{1}{v-u}\left[F(v)-F(u)-F^{\prime}(t)(v-u)\right] \in B_{\epsilon} \subseteq V$ or $F(v)-F(u)-F^{\prime}(t)(v-u) \in$ $(v-u) V$ whenever $t \in[u, v] \cap E \subseteq[a, b] \cap E$ and $|v-u|<\delta$. This shows that $f$ is $\mathcal{D}^{*}$ integrable.

We point out that the difficulty in showing the converse of Theorem 13, if it were true, lies in showing that $A C^{*}$ in the sense of Definition 4 implies $A C^{*}$ in the sense of Definition 9. Indeed, if the norm of the sum of vectors is strictly smaller than some positive number, the sum of the norms of the vectors cannot be forced to be strictly smaller than the same positive number. It seems that a weaker version of the $\mathcal{D}_{*} \mathcal{B}$-integral for Banachvalued functions (possibly not yet defined) may be equivalent to the $\mathcal{D}^{*}$-integral. This still remains to be investigated and seen.

Solodov in [13] gave a characterization of the strong Henstock integral using $A C G^{*}$ functions (the Denjoy-Bochner integral). The next result is somehow related to that work of Solodov. However, as our example will show, the converse of this result is not true. Further, note that this result is immediate from Theorem 9 and Theorem 12.

Theorem 14. If $f:[a, b] \rightarrow X$ is Denjoy integrable on $[a, b]$, then it is $S H$ integrable on $[a, b]$.

Example 3. To see that the converse of Theorem 9 and Theorem 14 are not true, consider the space $\mathcal{F}[0,1]$ of all real-valued functions on $[0,1]$. We will construct a separated family of semi-norms on $\mathcal{F}[0,1]$ from which a locally convex topology on $\mathcal{F}[0,1]$ exists (see [10]). For each $\alpha \in[0,1]$, let $\rho_{\alpha}(f)=|f(\alpha)|$ for all $f \in \mathcal{F}[0,1]$ and let $\mathcal{P}=\left\{\rho_{\alpha}: \alpha \in[0,1]\right\}$. For $f, g \in \mathcal{F}[0,1]$ and $c \in \mathbb{R}, \rho_{\alpha}(f+g)=|f(\alpha)+g(\alpha)| \leq|f(\alpha)|+|g(\alpha)|=\rho_{\alpha}(f)+\rho_{\alpha}(g)$ and $\rho_{\alpha}(c f)=|c f(\alpha)|=|c| \rho_{\alpha}(f)$. If $\rho_{\alpha}(f)=0$ for all $\alpha \in[0,1]$, then $f$ is the zero function. Hence, $\mathcal{P}$ is a separating family of semi-norms. In this space, the set $V_{\alpha, n}=\{x \in \mathcal{F}[0,1]$ : $\left.\rho_{\alpha}(x)<\frac{1}{n}\right\}$ is an absorbing, balanced and convex $\theta$-nbd for $\alpha \in[0,1]$ and $n \in \mathbb{N}$. The finite intersections of sets of this form is a local base at $\theta$ for the topology on $\mathcal{F}[0,1]$ (see [10])). 
Next, define $h:[0,1] \rightarrow \mathcal{F}[0,1]$ by $h(t)=e_{t}$ where $e_{t}$ is a function on $[0,1]$ given by

$$
e_{t}(x)= \begin{cases}1, & \text { for } x=t \\ 0, & \text { for } x \neq t\end{cases}
$$

Consider the function $\Theta:[0,1] \rightarrow \mathcal{F}[0,1]$ that maps each number in $[0,1]$ to the zero function on $[0,1]$. Clearly, $\Theta$ is an $A C G^{*}$ function. Since $\Delta(V, \Theta, h) \subseteq \Delta(U, \Theta, h)$ whenever $U \subseteq V$, it is enough to prove that $\Delta(U, \Theta, h)$ has measure zero for any local base $U$ at $\theta$ to show that $h$ is weak Denjoy integrable (hence, also $S H$-integrable by Theorem 12). Now, given a local base $U$ at $\theta, U$ is a finite intersection of some sets of the form $V_{\alpha_{i}, n_{i}}$ for $1 \leq i \leq k$. Let $\beta \in[0,1]$. If $\beta$ is distinct from $\alpha_{i}$ for each $1 \leq i \leq k$, then we may choose $\delta>0$ to be sufficiently small so that $\alpha_{i} \notin(\beta-\delta, \beta+\delta)$ for each $1 \leq i \leq k$. Then

$$
\rho_{\alpha_{i}}(\Theta(v)-\Theta(u)-h(\beta)(v-u))=0
$$

whenever $0 \leq u \leq \beta \leq v \leq 1$ with $v-u<\delta$ and $1 \leq i \leq k$. This certainly implies that $\beta \notin \Delta(U, \Theta, h)$. So, $\Delta(U, \Theta, h) \subseteq\left\{\alpha_{i}: 1 \leq i \leq k\right\}$ and $\Delta(U, \Theta, h)$ has a measure zero. Therefore, $h$ is weak Denjoy integrable with weak Denjoy primitive $\Theta$. By Theorem 5, observe that

$$
[0,1]=\bigcup_{\alpha \in[0,1], n \in \mathbb{N}} \Delta\left(V_{\alpha, n}, \Theta, h\right) \subseteq \bigcup_{\theta-n b d U} \Delta(U, \Theta, h) \subseteq[0,1]
$$

is the set at which the derivative of $\Theta$ does not exist. Thus, $h$ is not Denjoy integrable.

\section{Conclusion}

Although it is likely that the $H K$ and the $S H$ integrals coincide, showing the possible equivalence is not the focus of this present paper. We thus leave to the interested readers the task of showing whether or not these integrals are equivalent.

In this paper, $A C^{*}$ and $A C G^{*}$ properties have been introduced for LCTVS-valued functions. The $A C G^{*}$ property together with the concepts of differentiability and $\Delta(U, F, f)$, where $U$ is a $\theta$-nbd and $F$ and $f$ are LCTVS-valued functions, have been used to define two Denjoy-type integrals. When $X$ is a Banach space, the Denjoy-Bochner integral defined by Solodov is included in the Denjoy integral. It shown that these Denjoy-type integrals are included in the $S H$-integral. However, as shown in the paper, there exists a weak Denjoy integrable (also an $S H$-integrable) function which is not Denjoy integrable. It may be worthwhile to investigate whether or not the converse of Theorem 12 is true. The authors conjecture that the converse of that result is not true. 


\section{Acknowledgements}

We would like to extend our heartfelt thankfulness to the referee for taking the time to read the initial manuscript and for the comments and suggestions that led us to this improved version of the paper. Further, we would like to thank the Department of Science and Technology (DOST), Phillipines, and MSU-Iligan Institute of Technology for funding this research.

\section{References}

[1] S. Canoy Jr. and M. Navarro. A Denjoy-type integral for Banach-valued functions. Rend. Circ. Mat. Palermo, 44(2):330-336, 1995.

[2] S. Cao. The Henstock Integral for Banach-Valued Functions. Southeast Asian Bull. Math., 16(1):35-40, 1992.

[3] J. Dugundji. Topology. Allyn and Bacon, Inc., 470 Atlantic Avenue, Boston, USA, 1966.

[4] A. Khintchine. Sur le procede d'integration de M. Denjoy. Mat. Sbornik, 30:548-557, 1916.

[5] N. Lusin. Sur les proprietes de l'integrale de M. Denjoy. Comptes Rendus De l'Academie des Sciences, 155:1475-1478, 1912.

[6] R. Maza and S. Canoy Jr. On SL-integral of LCTVS-valued Functions. Real Analysis Exchange, 46(2):505-522, 2021.

[7] R. Paluga. Absolute Continuity in Topological Vector Spaces. Matimyas Matematika, 27(3):42-46, 2004.

[8] R. Paluga and S. Canoy Jr. The Henstock Integral in Topological Vector Spaces. Matimyas Matematika, 24(3):34-47, 2001.

[9] R. Paluga and S. Canoy Jr. On the Strongly Henstock integral in Topological Vector Spaces. Journal of Research in Science and Engineering, 1(3):46-50, 2004.

[10] W. Rudin. Functional Analysis. McGraw-hill, Inc., Singapore, second edition edition, 1991.

[11] H. Schaefer. Topological Vector Spaces. Springer-Verlag, New York Heidelberg Berlin, 1971.

[12] V. Skvortsov and A. Solodov. A variational integral for Banach-valued functions. Real Analysis Exchange, 24(2):799-805, 1998-1989.

[13] A. Solodov. A Riemann-type definition for the restricted Denjoy-Bochner integral. Fundam. Prikl. Mat., 7(3):887-895, 2001. 
[14] L. Yee and R. Výborný. Integral: An Easy Approach after Kurzweil and Henstock. Cambridge University Press, New York, 2000.

[15] L.P. Yee. Lanzhou Lectures on Henstock Integration, volume 2 of Series in Real Analysis. World Scientific, NUS, Singapore, 1989.

[16] L.P. Yee. On $A C G^{*}$ Functions. Real Analysis Exchange, 15(2):754-759, 2001. 